\title{
Observations of Polar Cusp and Polar Cap Ionospheric Irregularities and Formation of Ionospheric Holes Using Topside Sounder on board EXOS-C (Ohzora) Satellite
}

\author{
Takahiro OBARA ${ }^{1}$ and Hiroshi OYA ${ }^{2}$ \\ ${ }^{1}$ Institute of Space and Astronautical Science, 3-1-1, Yoshinodai, Sagamihara, Kanagawa 229, Japan \\ ${ }^{2}$ Geophysical Institute, Tohoku University, Aoba, Aramaki, Sendai 980, Japan
}

(Received December 5, 1988; Revised August 19, 1989)

A topside sounding experiment by the PPS system on board the EXOS-C (Ohzora) satellite made successful observations in the polar ionosphere. From analyses of the ionograms the following results have been obtained.

i) Associated with the particle precipitation into the polar cusp region, small scale irregularities causing the spread $F$ phenomena with electron density enhancement have been generated.

ii) In the polar cap ionosphere, there are also small-scale irregularities of the electron density causing the spread $F$ phenomena. These irregularities have also been associated with an enhancement of the electron density. The origin of these irregularities in the polar cap is in the polar cusp region, and the irregularities are transported into the polar cap region by convection from the cusp region.

iii) In the midnight polar cap region, an electron density depression region, called a plasma hole, has been sometimes observed. The magnetic activity index corresponding to detection of the plasma hole always indicates a very low value. This is interpreted to occur as the result of a reduced plasma convection in the polar cap region. While the polar cap plasma escapes continuously due to the effect of the polar wind and losses through the attachment process, the supply is insufficient at the ionospheric level with the lack of convection, causing the plasma hole.

\section{Introduction}

Studies of the irregular structure of the polar cusp and polar cap ionospheres provide us with the clue for understanding dynamical processes occurring in the polar ionosphere. For this purpose topside sounder experiments have contributed by investigating the irregularities in the ionosphere as well as sounding the vertical structure of the ionosphere. Using the topside sounding data by Alouette and ISIS, PERTIE (1963) have indicated that there were various kinds of irregularities in the polar ionosphere and that the spread $F$ echoes shown in the ionograms are manifestation of the small-scale irregularities. DYSON and WINNINGHAM (1974) have pointed out that these small-scale irregularities expand up to the altitude range of about $3000 \mathrm{~km}$ in the polar cusp region. They have inferred that these irregularities were made by soft particle precipitation into the polar cusp ionosphere. 
However, detailed study on the spread $F$ phenomena in the polar cap ionosphere has been deferred until very recently because of the lack of sounding satellite tracking stations in very high latitude regions.

The plasma hole was first found by the AE-C satellite using an ion-mass spectrometer (BRINTON et al., 1978). The authors inferred that the plasma hole is made by the influence of plasma convection in the polar cap. Though the plasma hole has the tendency to appear in magnetically quiet periods, a detailed formation mechanism as well as the structure of the vertical plasma distribution associated with the hole have not been clarified yet.

The PPS instrument in the ninth Japanese scientific satellite EXOS-C (Ohzora) that was launched on February 14, 1984 has done experiments for studies on the plasma conditions and wave particle interaction processes in the ionosphere of global extent, including the polar region and the Brazilian anomaly of the magnetic field. The system and initial results of the PPS system on board the EXOS-C (Ohzora) satellite have been reported in previous papers (OYA et al., 1985; OBARA and OYA, 1985). In this paper the PPS results for the plasma turbulences in the polar cusp and polar cap are reported as being concentrated on the observation of the spread $F$ phenomena and for the polar cap plasma distribution corresponding to the polar cap plasma hole phenomena. Studies are especially focused on:

1) the generation mechanism of the spread $F$ irregularities related to the precipitating particles in the cusp region,

2) observation of the polar cap spread $F$ phenomena, related to high latitude plasma convection, and

3) observation of the polar cap plasma hole, related to high latitude plasma convection.

\section{Polar Cusp Spread $F$}

Largely enhanced spread $F$ phenomena were discovered in the cusp region by the Alouette I satellite experiments (PERTIE, 1963). Because of the scattering of the radio waves emitted from the satellite, it was concluded that each irregularity must have a wave length equal to one half of the radio wavelength. Though there was understanding that these small-scale irregularities were caused by the particle precipitation in the cusp region, a complete explanation for the generation mechanism of these irregularities has not been made yet.

Typical examples of a series of ionograms revealing the spread $F$ phenomena are indicated in Fig. 1; the results were obtained by a topside sounding experiment of the PPS on board the EXOS-C (Ohzora) satellite, while the satellite EXOS-C passed through the cusp region. In the early stage of the observation of this sequence, the spread $F$ phenomena were evidently not enhanced; only slight spread echoes are visible in the lower level of the topside ionosphere (see ionograms from (a) to (b)). As the satellite moved to the dayside polar cusp region from the polar cap region, however, the spread $F$ phenomena became very intense (see ionograms from (b) to $(\mathrm{g})$ ). When the satellite moved in the region (about $71^{\circ}$ invariant latitude), the 
EXOS-C PPS 1985.5.14 3:13:45-3:19:04

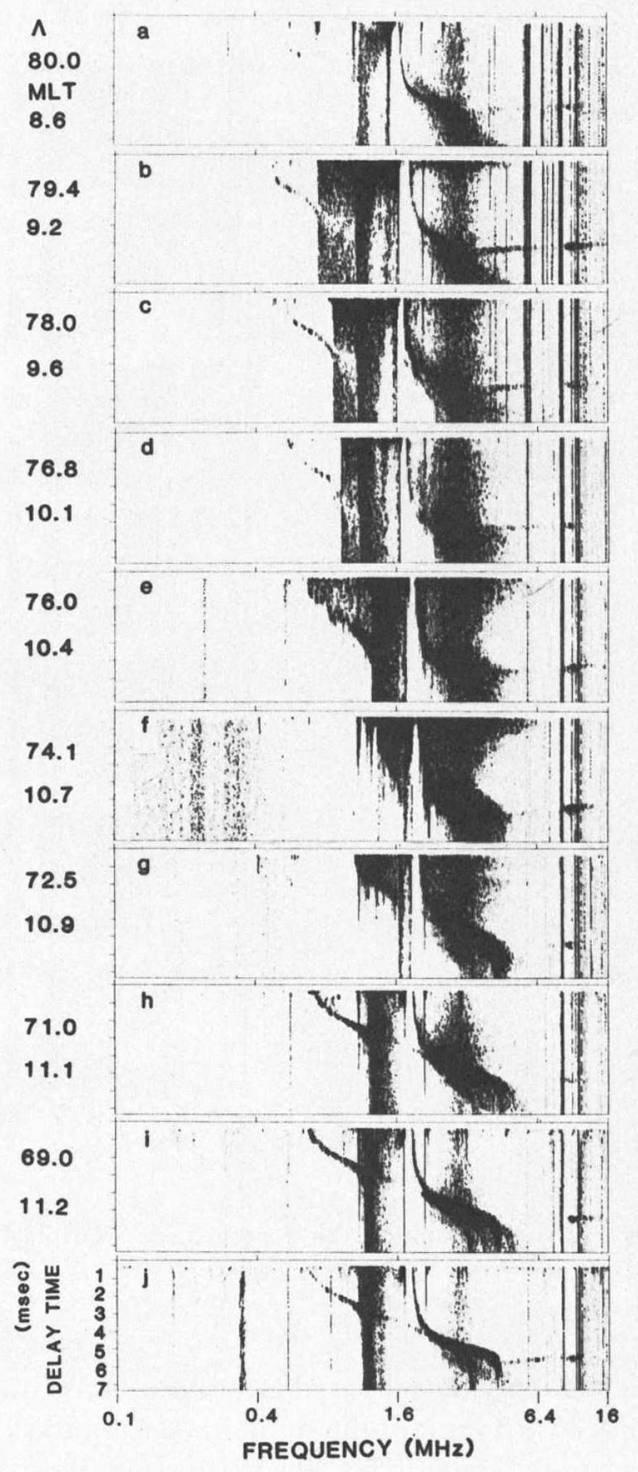

Fig. 1. EXOS-C (Ohzora) ionograms observed from 03:13:45 to 03:19:04 UT on May 14, 1985 when the satellite passed through the cusp region. The invariant latitude $(\Lambda)$ and magnetic local time (MLT) are indicated for each ionogram. Time delay unit is indicated only in the bottom panel, but is the same for all panels. 


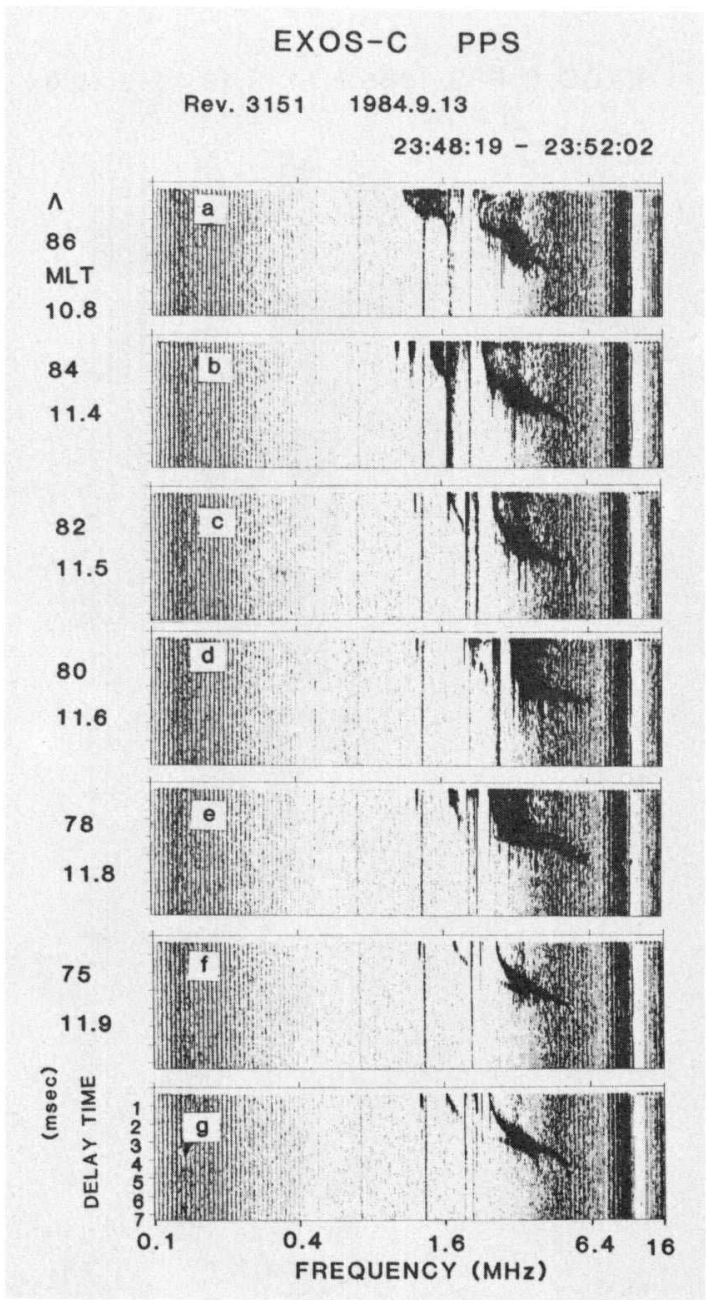

Fig. 2. Same format as Fig. 1. Observations have been made from 23:48:19 to 23:52:02 UT on September 13, 1984.

spread $F$ phenomena suddenly disappeared (see ionograms (h), (i), $\cdots \cdots$ ); the result suggests that the equatorward limit of the small scale irregularity region indicated in the form of the spread $F$ phenomena has a very abrupt edge.

In Figs. 2 and 3, different cases of the enhanced spread $F$ phenomena across the polar cusp region have been indicated. Between about $80^{\circ}$ and $78^{\circ}$ invariant latitude, for the case of Rev. 3151, spread $F$ phenomena indicating small-scale irregularities have been found up to the satellite height of $500 \mathrm{~km}$. As the satellite moved to the lower latitude side, the spread $F$ phenomena abruptly disappeared (see 


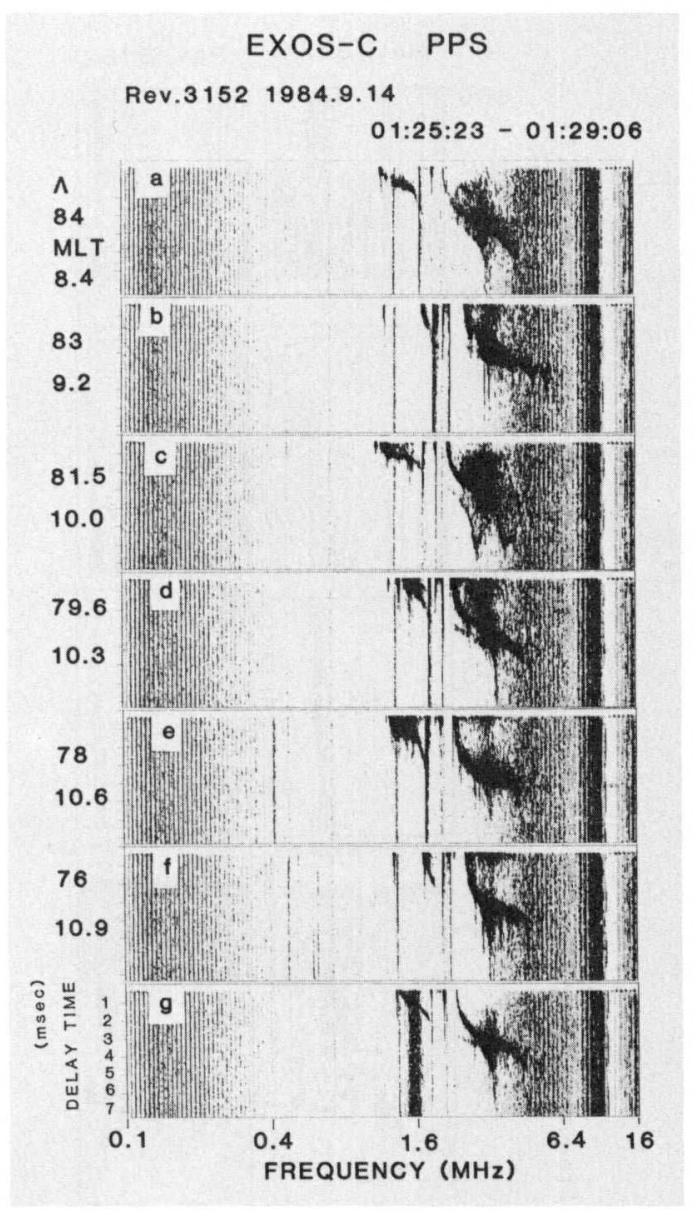

Fig. 3. Same format as Fig. 1. Observations have been made from 01:25:23 to 01:29:06 UT on September 14, 1984.

ionogram (f)). The data observed in the case of Rev. 3152 also show intense spread $F$ phenomena between $83^{\circ}$ and $78^{\circ}$ invariant latitude, corresponding to the polar cusp region.

On the EXOS-C (Ohzora) satellite, a particle detector system ESP (Energetic Spectrum of Particles) has been installed (MUKAI et al., 1985). The energetic particle observation data have been expressed in the form of an energetic spectrum versus the observation time; i.e., in the form of an $E$ - $t$ diagram (MUKAI et al., 1985). In Fig. 4 , local time surveys of the $E$ - $t$ diagrams of the electrons and ions within the polar region have been presented for both Rev. 3151 and Rev. 3152. In Fig. 4, the top two panels (LE) indicate the low energy electron flux in a range from $10 \mathrm{eV}$ to $300 \mathrm{eV}$, while the second two panels (HE-3) express the high energy electron flux in the 


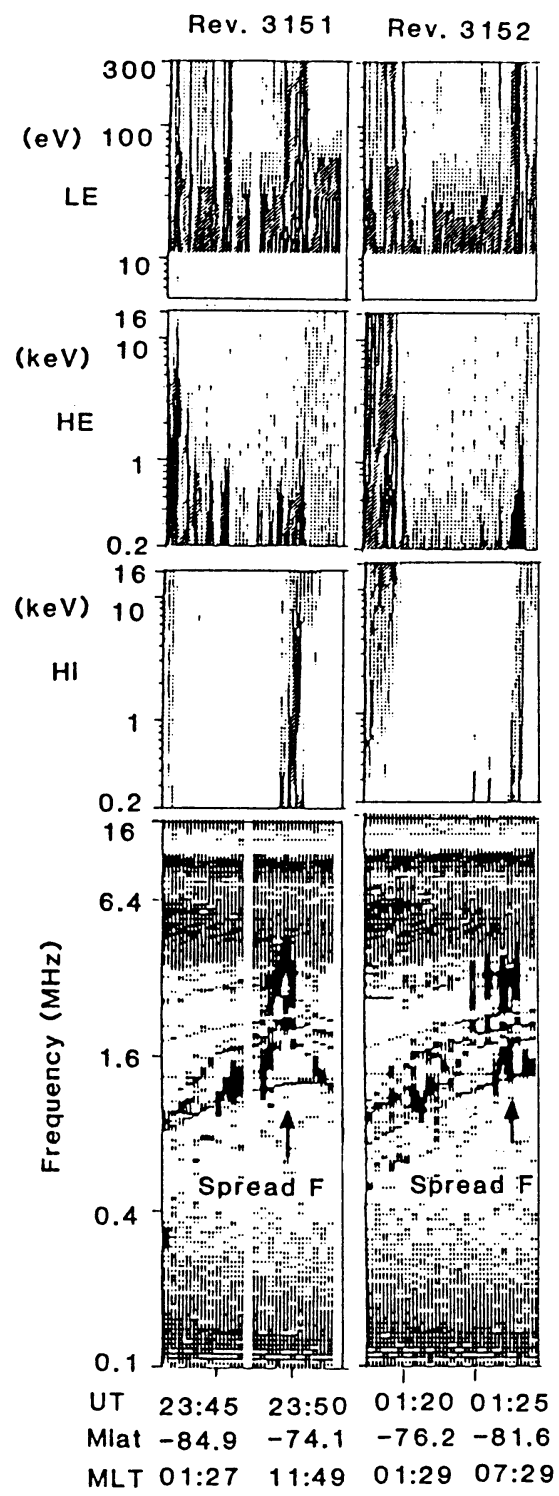

Fig. 4. Comparison of the $E$ - $t$ diagrams of the energetic particles (ESP) and the $f-t$ plot of the plasma wave sounder (PPS) for two succeeding paths. Corresponding to the regions of enhanced particle precipitation, the spread $F$ phenomena have been seen centered around the period given by arrows. 
range from $0.2 \mathrm{keV}$ up to $16 \mathrm{keV}$. The ion flux (HI) is expressed in the third two panels in Fig. 4 , indicating an energy range from $0.2 \mathrm{keV}$ to $16 \mathrm{keV}$. These particle data indicated that there was very intense precipitation of energetic particles in the cusp region. The particle events correlated with HI precipitation were clearly seen at UT 23:50 and 01:27, respectively, in Rev. 3151 and Rev. 3152. The dominant precipitating particles are in the range of about a few hundred $\mathrm{eV}$ to a few $\mathrm{keV}$ for ions. The lowest two panels in Fig. 4 show the observation data of the topside sounder experiment expressed in the form of a dynamic spectrum whose ordinate and abscissa indicate the frequency of the detected echoes and the observation time, respectively. In the diagram in the bottom panels, the intensity indicates the received signal level of the natural plasma waves as well as the radio waves corresponding to the transmitted RF pulse. In the lowest panel of Fig. 4, the arrows indicate the portions where the spread $F$ phenomena are enhanced. That is, the dark portions in the frequency range 3 to $5 \mathrm{MHz}$ corresponding to the spread $F$ phenomena show that the spread $F$ irregularities coincide with the particle precipitation in the polar cusp ionosphere, suggesting that the generation of spread $F$ irregularities in the polar cusp ionospheric plasma are caused by the beam-plasma interaction processes. The observation regions of these cases have been plotted in Fig. 5 as a polar map, where the regions of occurrence of the spread $F$ phenomena have been marked with thick lines on the satellite paths. The results suggest that the spread $F$ phenomena

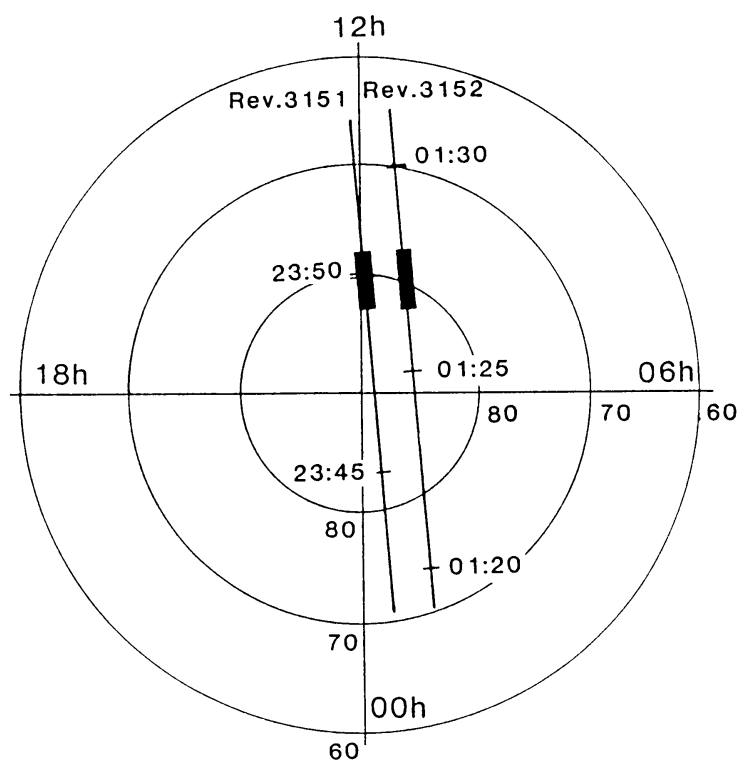

Fig. 5. Satellite paths for Rev. 3151 and Rev. 3152 of the EXOS-C observations, projected on the polar map. In each path, enhancement of particle precipitation associated with the spread $F$ phenomena, in the cusp region, is indicated by thick lines. 
are stretched in a longitudinal direction persisting for a few hours, at least, in the cusp region.

From the data obtained in Rev. 3151 and Rev. 3152, it is also clarified that the plasma density is largely enhanced in the region where the flux of the precipitating particles is enhanced (see Fig. 4). At the same time as the ionization enhancement, progress of the plasma instabilities that become the origin of the spread $F$ phenomena are enhanced by the particle precipitation.

\section{Polar Cap Spread $F$}

In the high latitude region, spread $F$ phenomena have been observed in a wide range of areas covering from the auroral oval region to the polar cap region (see Fig. 6). Example records of spread $F$ observed in the polar cap (including the polar cusp) are given in Figs. 7 and 8 for the Rev. 3122 and Rev. 3123 observations respectively of the EXOS-C (Ohzora) satellite. In Rev. 3122 (see Fig. 7) we can see remarkable variation of the occurrence of the spread $F$ phenomena with evident enhancement in the panels from (d) to (q). Also, in Rev. 3123 (see Fig. 8), the regions enhancement of the spread $F$ can be identified in the ionograms from (d) to (k) corresponding to the invariant latitude from $81^{\circ}$ to $73^{\circ}$. In these cases the equatorward limit of the polar cusp irregular region also shows an abrupt edge, and the electron density shows a higher value in the cusp region than the region surrounding the cusp. The

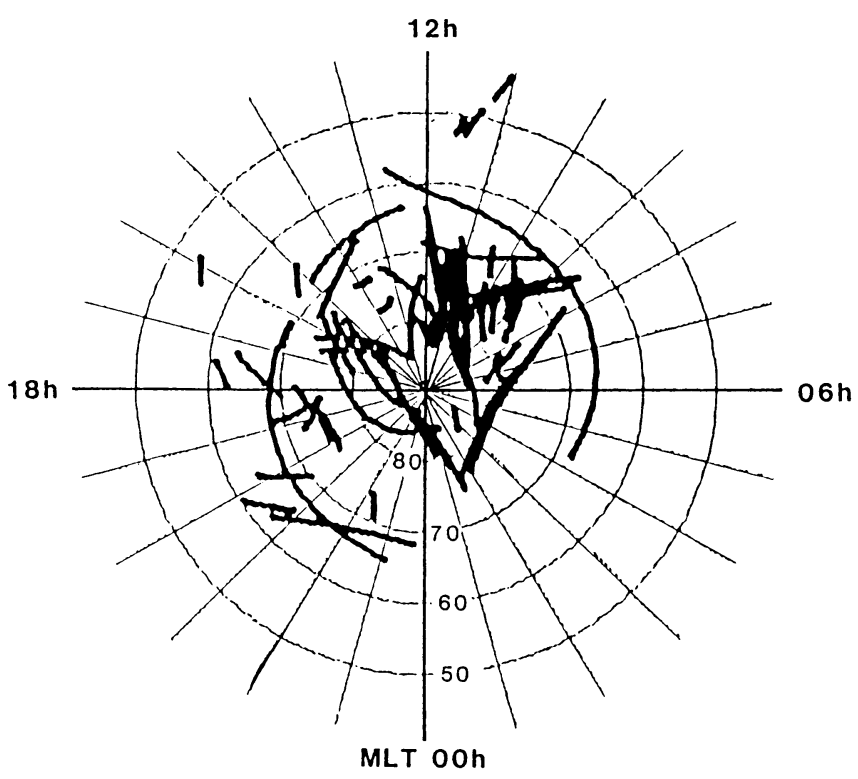

Fig. 6. Plots of the observation region of the spread $F$ phenomena detected by the EXOS-C observations in the polar region, projected on the polar map. 
EXOS-C PPS 1984.9.11 01:05:10-01:15:13

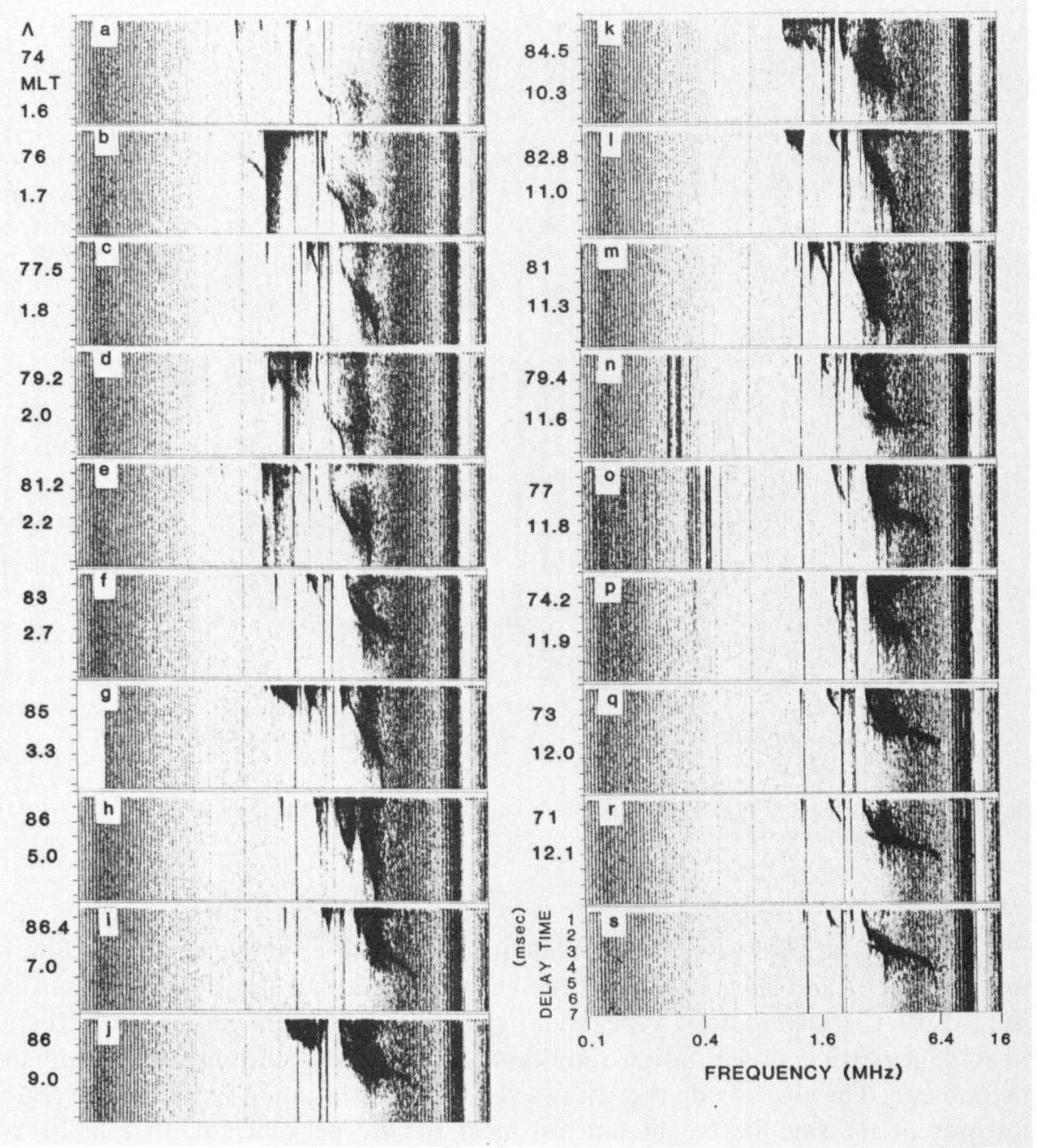

Fig. 7. Ionograms observed by the satellite EXOS-C from 01:05:10 to 01:15:13 UT on September 11, 1984, passing through the polar cap region; ionograms from (n) to (q) corresponding to exact cusp region. The format is same with Fig. 1.

crossing of the polar cusp spread $F$ regions as given in the case of Rev. 3151 from panel (b) to (e), in Fig. 2, and also from (b) to (e) for Rev. 3152, in Fig. 3, correspond to the portions of the series of ionograms from (n) to (q) in Rev. 3122 (Fig. 7) and ionograms from (h) to (k) in Rev. 3123 (Fig. 8). 


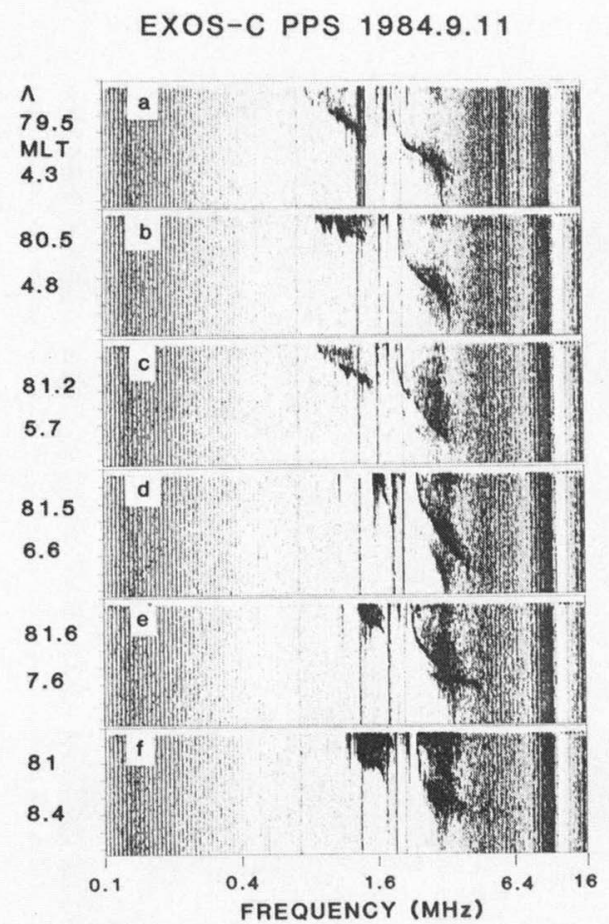

$02: 44: 50-02: 51: 13$

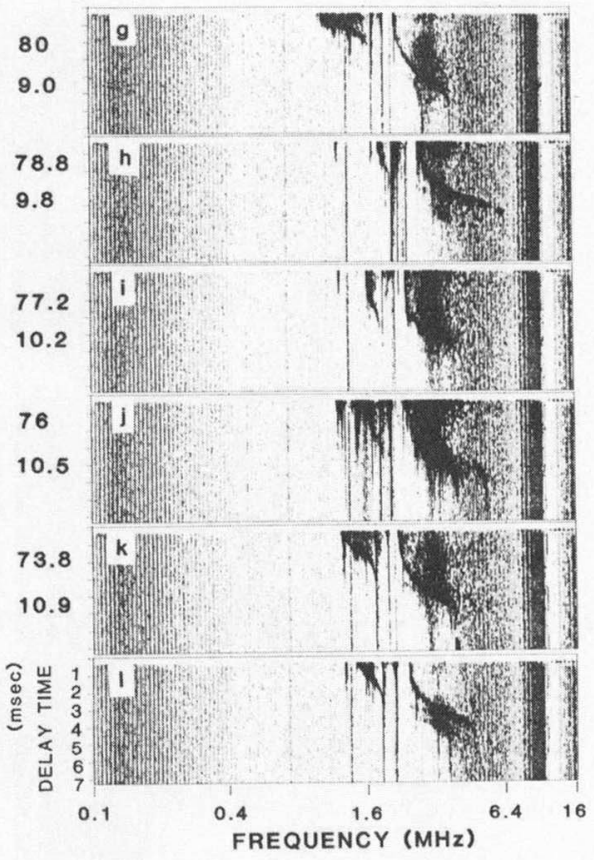

Fig. 8. Same format as Fig. 7. Observations have been made from 02:44:50 to 02:51:13 UT on September 11, 1984.

Particle precipitation is quite evident in the cusp crossing of the satellite for both Rev. 3122 and Rev. 3123 as given in the $E$ - $t$ diagrams of the energetic particles (see Fig. 9). Corresponding to the polar cap spread $F$ phenomena, however, there is no evident particle precipitation simultaneous with the occurrence of the spread $F$ phenomena. The electron density shows very large variation in the polar cap region, not only at the satellite height but also near the $F 2$ peak height. In Fig. 10, two succeeding satellite paths, Rev. 3122 and Rev. 3123, are projected on the polar map, where the PP's indicate regions of particle precipitation and the SF's indicate the regions of spread $F$ observations. The SF regions are evidently spread in ranges wider than the particle precipitation regions.

This large expansion of the region of occurrence of polar cap spread $F$ phenomena is largely correlated with the magnetic activity index ( $K p$ index) as given in Fig. 11, where one week of the $K p$ value is plotted including the observations periods of Rev. 3122 and Rev. 3123. In Fig. 11, the occurrence of the spread $F$ phenomena is marked with "A", "B", and "C". Satellite paths with spread $F$ phenomena in the polar cusp region were indicated with " $\mathrm{B}$ ", and the cases where 


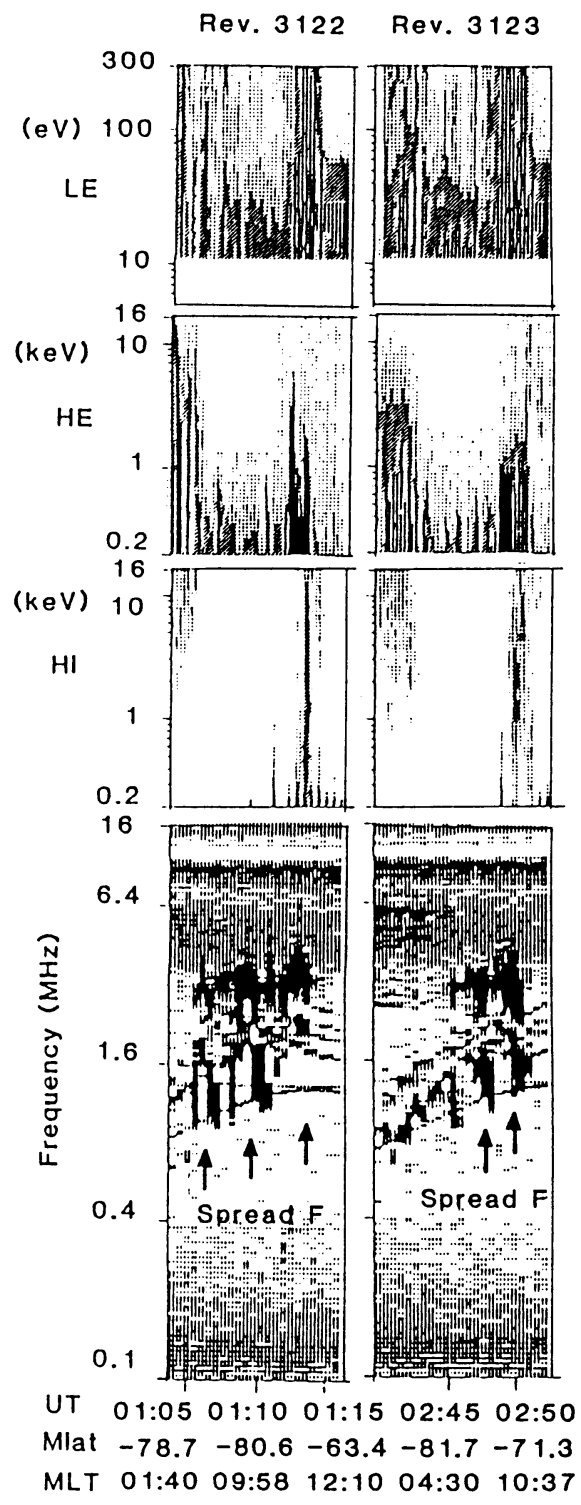

Fig. 9. Comparison of the energetic particle data and the plasma wave sounder results of the EXOS-C observations in the polar cap regions. There are spread $F$ phenomena (see arrows) even in the region of no particle precipitation, while there are well-correlated regions of the spread $F$ phenomena with particle precipitation in the cusp region. 


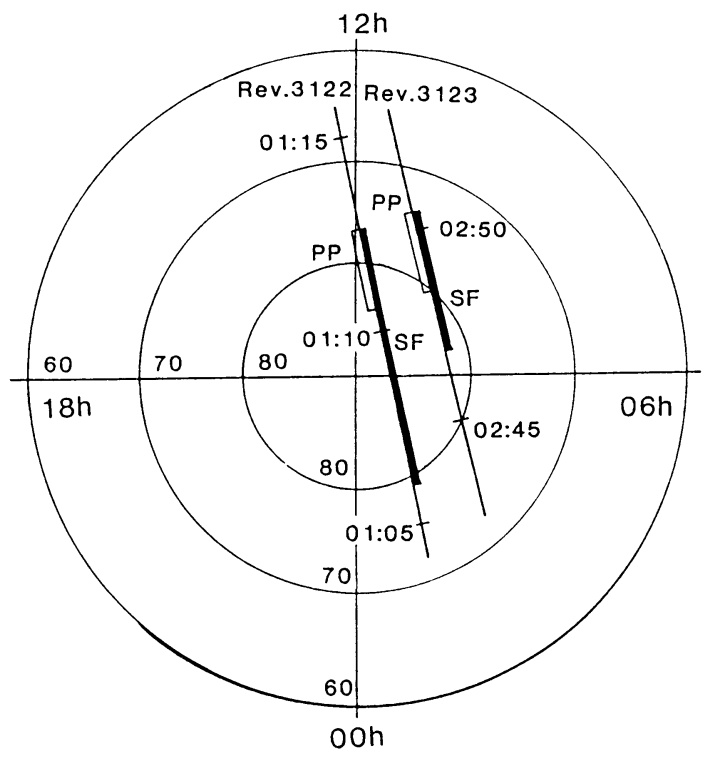

Fig. 10. Polar plots of satellite paths for Rev. 3122 and Rev. 3123. In each path, the region where the satellite observed spread $F$ phenomena is indicated with a thick line; the appendix line is added for the portion where particle precipitation was detected.

History of $\mathrm{Kp}$ Index

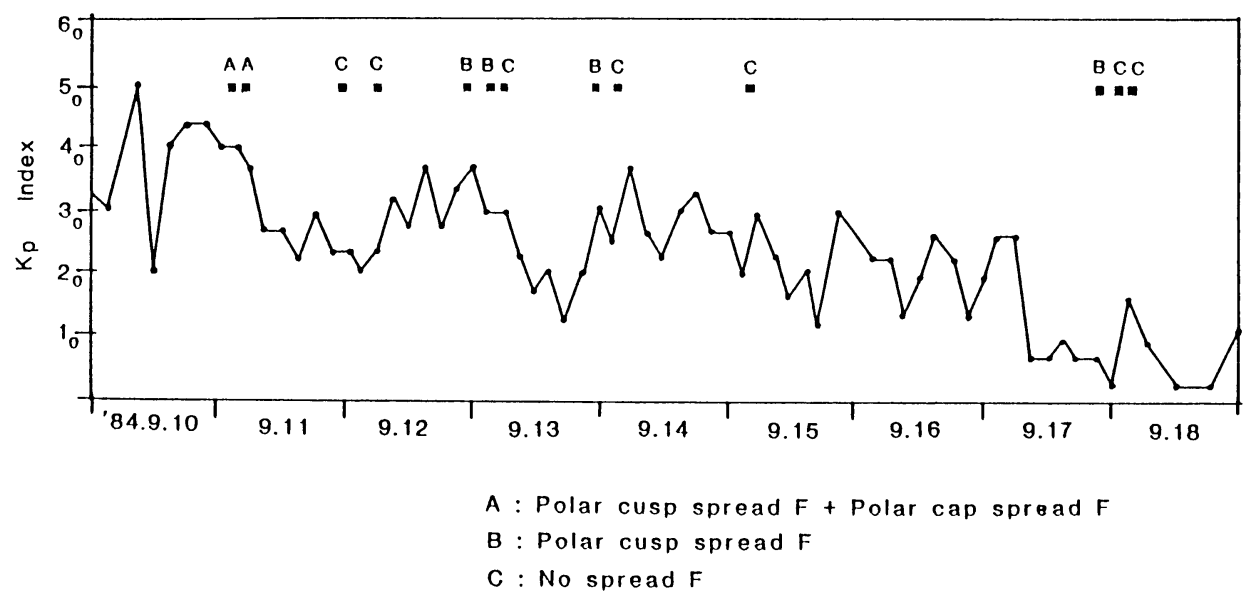

Fig. 11. The $K p$ index for one week from September 10, 1984 to September 18, 1984, including the period when the polar cap spread $F$ phenomena have been observed. It is evident that the observations of the polar cap spread $F$ phenomena were made in a period of very high $K p$ index, while there were no observations of the spread $F$ in the polar cap region ionosphere in cases of low $K p$ values. 
the spread $F$ phenomena were found both in the polar cap and the polar cusp ionospheres, as in Figs. 7 and 8, are given by " $A$ ". Mark " $C$ " corresponds to the case of no spread $F$ phenomena. We can see, then, that cases of $\mathrm{A}$ and $\mathrm{B}$ occur in the period of high $K p$, while the $K p$ index always shows a low value for the situation marked C.

It is well known that the high latitude ionospheric convection is enhanced when the magnetic activity becomes large. We can infer, then, that the polar cap spread $F$ phenomena originate in the region of the polar cusp and make convection into the polar cap region, considering the high $K p$ index value $\left(K p=4_{0}\right)$ for the occurrence of case A. In Fig. 12 the plasma drift configuration with the auroral oval has been indicated; the anti-sunward plasma convection in the polar ionosphere is consistent with this hypothesis.

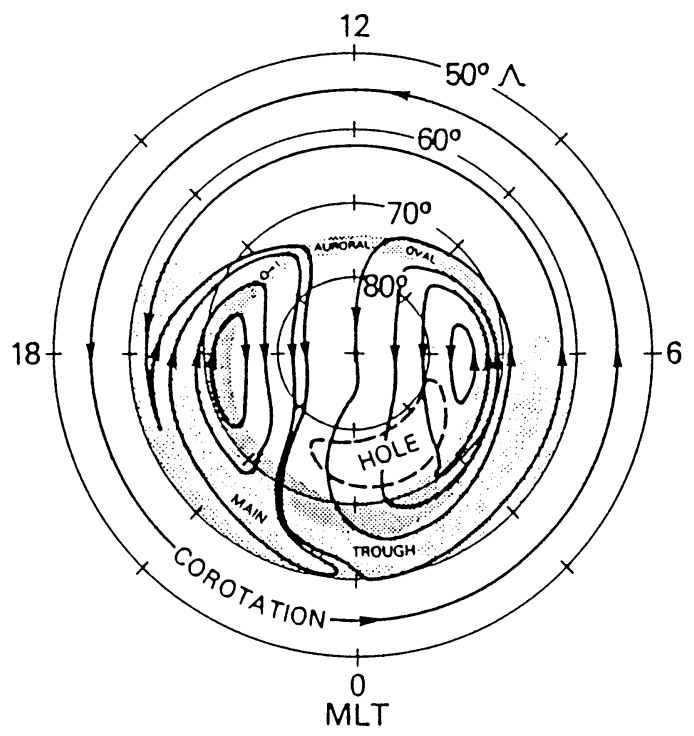

Fig. 12. Possible plasma drift stream lines in the polar cap ionosphere including the dayside auroral oval region; the anti-sunward drift is inferred to be enhanced in the case of the high magnetic activity (after BRINTON et al., 1978).

\section{Polar Cap Plasma Hole}

When the satellite is located within the nightside polar cap region, the low density region of the ionospheric plasma, called the plasma hole (BRINTON et al., 1978), has frequently been identified. In the succeeding ionograms from (a) to (e) shown in Fig. 13, a typical example of plasma hole crossing is indicated; ionogram (c) in Fig. 13 indicates the center of the hole showing the lowest plasma resonance 


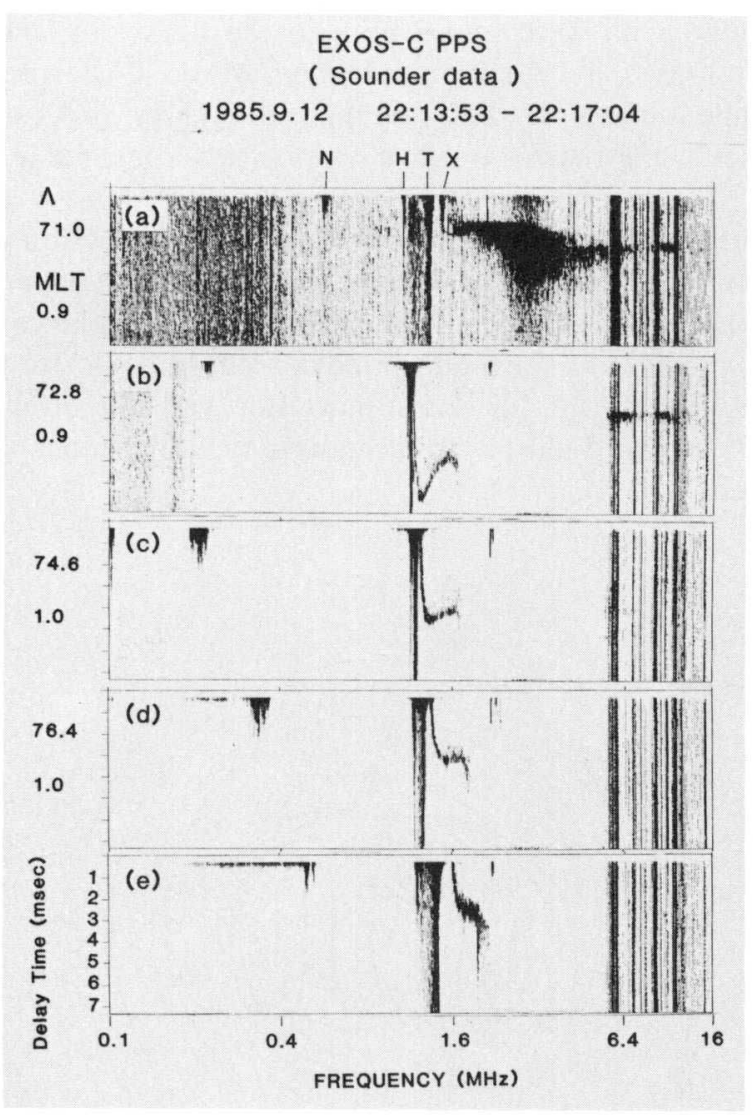

Fig. 13. EXOS-C (Ohzora) ionograms obtained as the satellite passed through the plasma hole region. The invariant latitude and the magnetic local time are indicated for each ionogram. The time delay unit is indicated only in the bottom panel, but is the same for all the panels.

near $250 \mathrm{kHz}$, which corresponds to $770 / \mathrm{cc}$ at the satellite height $(390 \mathrm{~km})$. An electron density profile calculated from these succeeding ionograms has been given in Fig. 14, which indicates the vertical structure of the topside ionosphere in a very wide range, including the plasma hole region. The structure of the ionospheric plasma distribution in this region is characterized by a sudden decrease of the electron density, not only at the satellite level but in the whole range of the topside ionosphere covering the $F 2$ peak height. The occurrence of this hole corresponds to the previously identified hole region (BRINTON et al., 1978).

We have made a statistical study on the occurrence of the hole using the Ohzora satellite sounder data obtained in the September, 1985 and July, 1986 periods. The occurrence of hole regions, in which the electron density at the satellite level is less than $1900 / \mathrm{cc}$, has been plotted on the polar map in Figs. 15(a) and 15(b) for the data 


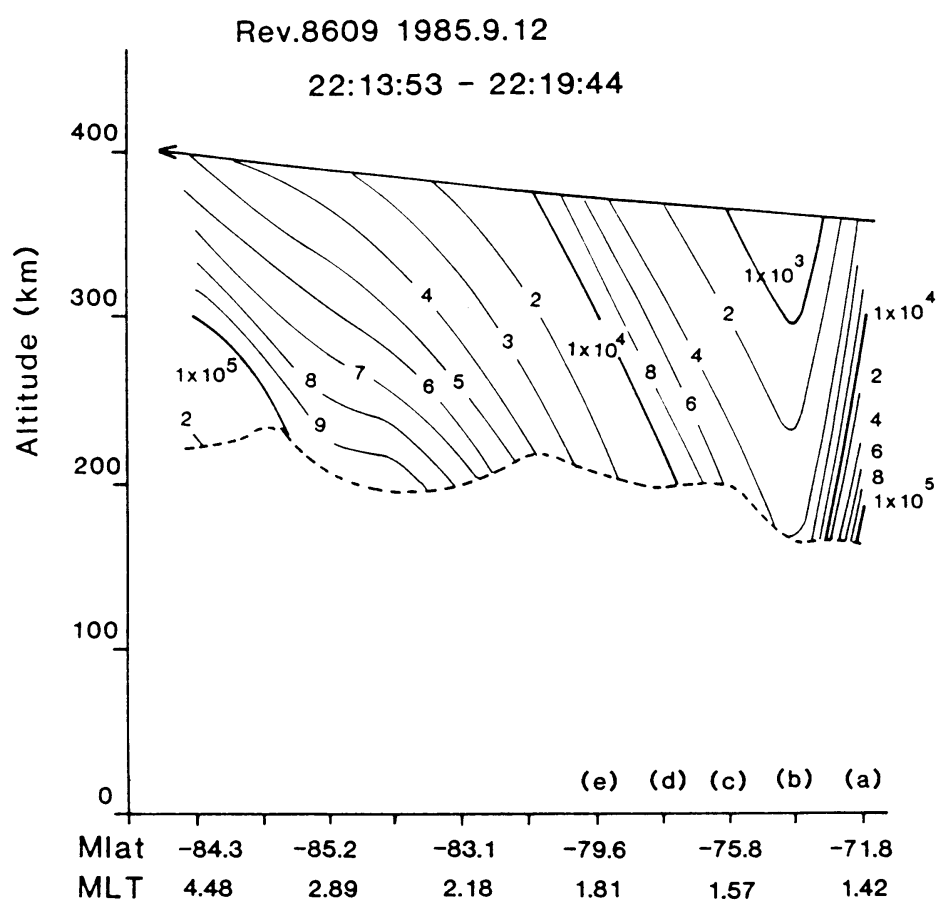

Fig. 14. Plasma density distribution given with equi-density contours of plasma density determined from the sounder ionograms as a function of height, geomagnetic latitude, and geomagnetic local time. The density unit is number of electrons $\mathrm{cm}^{-3}$ in this figure.

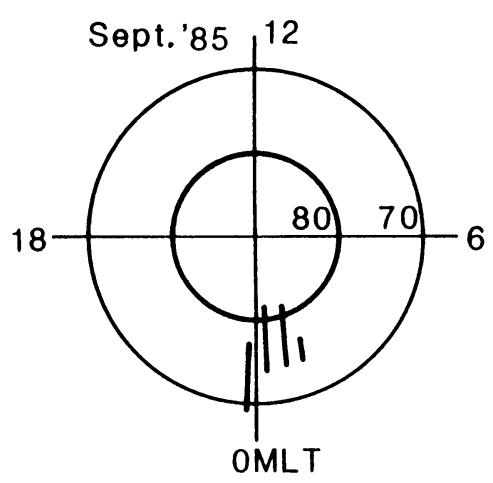

(a)

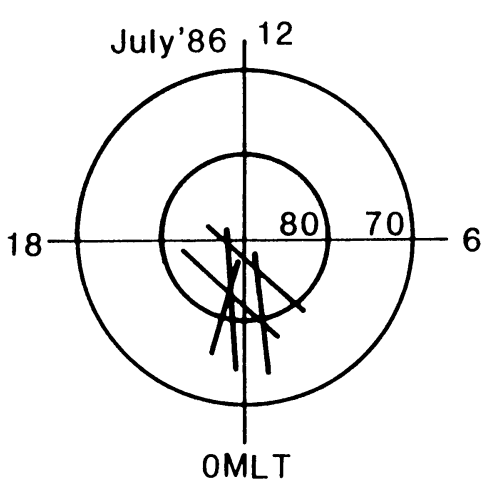

(b)

Fig. 15. Polar plots of the detected ionospheric holes, observed in September, 1985 (15(a)), and observed in July, 1986 (15(b)). 


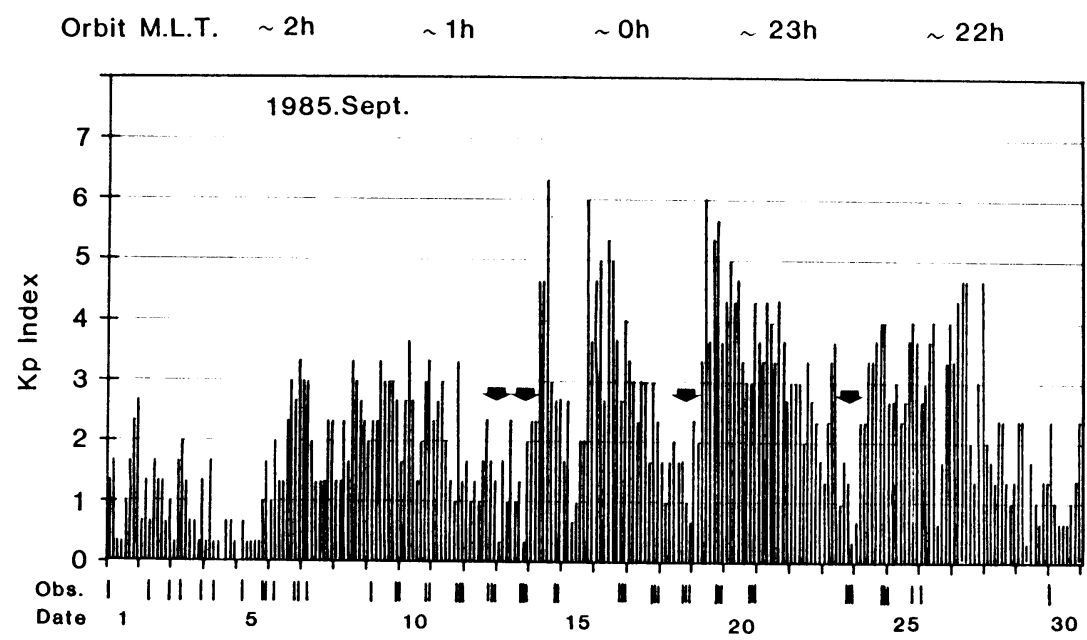

(a)

Orbit M.L.T.

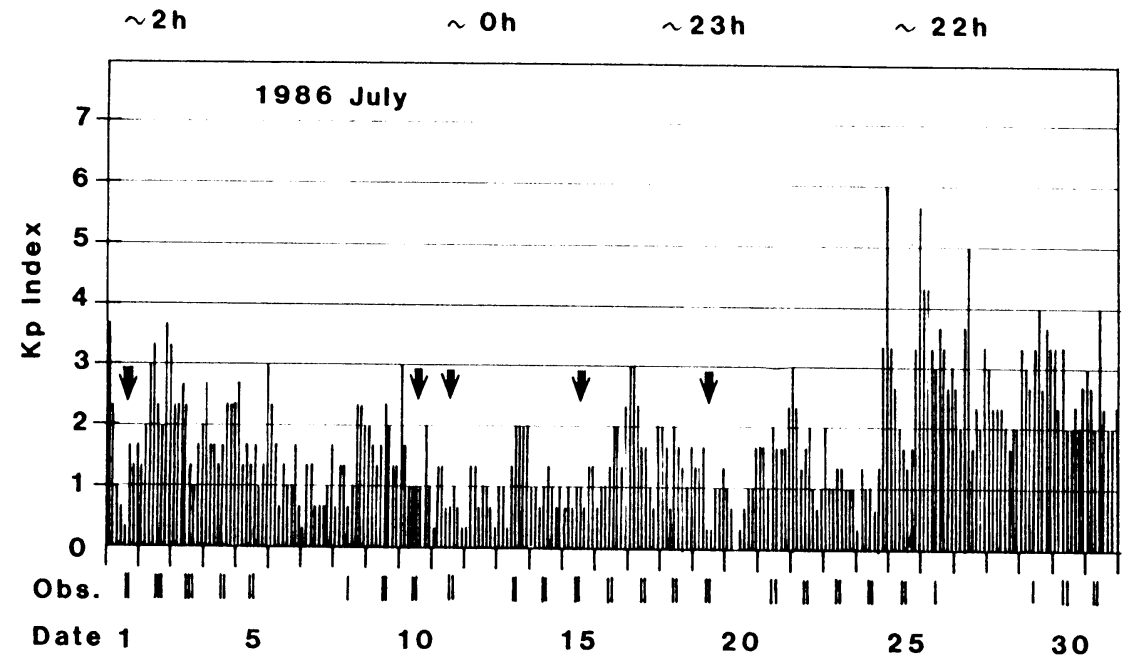

(b)

Fig. 16. History of $K p$ index variation in September, 1985 (16(a)) and in July, 1986 (16(b)). The periods when the plasma holes were observed are given by arrows; it is evident that the plasma holes are formed in the dead calm period of geomagnetic activity. Local time of the observation of the polar ionospheric regions are given in the top part of each figure. 
of September, 1985 and July, 1986 in the south polar regions. These polar plots of the hole region show remarkably that the hole regions are made in higher latitudes in the July period than in the September period; i.e., the hole position changes from a high latitude in the winter season to the low latitude side in springtime. Furthermore, the hole region is made in a wider range with a deeper and more tenuous density in the winter time. The magnetic activity index ( $K p$ index) is plotted in Figs. 16(a) and 16(b) for both observation periods, i.e., in the July and September periods, where the periods of the plasma hole observation are indicated by arrows. From these plots, it is evident that the ionospheric plasma hole appears only in a very calm state of the magnetic activity. As indicated at the top of Figs: 16(a) and 16(b), the magnetic local time of the polar cap crossing and detection of the plasma hole were made nearly at midnight meridian.

The maintaining of the topside ionosphere in the nightside polar cap region is mainly due to the transport of plasma from the dayside polar ionosphere by convection, which is directed from the noon to the midnight regions, as shown in Fig. 12. The formation of the hole therefore depends on the rate of supply of plasma by polar cap convection. In the magnetically calm state, the velocity of convection becomes weakened so that the supply of plasma into the nightside region becomes less effective. The formation of a deep hole is then caused as a result of plasma loss processes such as attachment and the polar wind effect, before receiving sufficient supply from the dayside ionosphere.

The source condition of the ionization, therefore, controls the formation of the hole as is the case of the plasma convection; this can be consistently understood from the seasonal dependency of the hole formation condition. In the winter, the entire polar cap region is in the nightside, where the ionization only relates to the precipitation of the energetic particles, while additional ionization due to solar UV radiation is added in the polar cap ionosphere in the spring. The difference of the hole formation conditions between the winter and spring periods given in Figs. 15(a) and $15(\mathrm{~b})$ is taken as a manifestation of this source effect.

\section{Conclusion}

From the analyses of the ionograms of topside sounding experiments (SPW experiments) with the PPS system on board the EXOS-C (Ohzora) satellite, the plasma irregularities in the polar cusp and polar cap ionospheres have been clarified. Associated with the particle precipitation into the polar cusp region, small-scale irregularities with large amplitude that causes the spread $F$ are generated. These results indicate that with growth of the irregularities, enhancement of the electron density takes place also in the cusp region. It is inferred that the enhancement of the topside ionospheric plasma density is caused by the precipitating particles. Though the relation between the plasma instabilities as the cause of the spread $F$ in the cusp and polar cap regions and the particle precipitation connected to the magnetosheath plasma is confirmed, studies on a detailed mechanism for generation of the density irregularities is deferred for future studies. 
As is the case of the cusp region, there are also small-scale irregularities of the electron density with large amplitude which causes the spread $F$ phenomena in the polar cap ionosphere. The irregularities in the polar cap do not, however, directly correspond to the particle precipitation. The present studies have indicated that these irregularities were originally generated in the polar cusp region and then are transported into the polar cap by the enhanced ionospheric convection.

In the polar cap region, the electron density depression region called the plasma hole has been detected associated with a dead-calm period of magnetic activity. Coincidence of the occurrence of the hole with dead calm conditions of the magnetic field shows that reduced plasma convection in the polar cap region becomes the cause of formation of the hole. The polar cap plasma is escaping continuously and reduced due to the effect of polar wind and attachment. When the ionized constituent is not supplied sufficiently, a deep hole is formed in the ionosphere level. Quantitative analysis for this evidence is, however, deferred to future studies.

The authors would like to express their sincere thanks to Prof. Itoh, manager of the EXOS-C (Ohzora) satellite, for current ISAS operation of the satellite; the authors would also like to express their thanks to all of the member of the EXOS-C and M-3S-4 teams of ISAS for successful launching and operation of the Ohzora (EXOS-C) satellite. The particle data in this paper are provided by ESP experiments onboard the Ohzora (EXOS-C) satellite; the authors are grateful to Drs. Mukai and Kaya for the use of the ESP data.

\section{REFERENCES}

Brinton, H. C., J. M. Grebowsky, and L. H. Brace, The high-latitude winter $F$ region at $300 \mathrm{~km}$ : Thermal plasma observation from AE-C, J. Geophys. Res., 83, 4767-4776, 1978.

Dyson, P. L. and J. D. Winningham, Topside ionospheric spread $F$ and particle precipitation in the dayside magnetospheric clefts, J. Geophys. Res., 79, 5219-5230, 1974.

Mukal, T., N. Kaya, H. Kubo, H. Matsumoto, T. Itoh, and K. Hirao, Initial observation of lowenergy charged particles by satellite Ohzora (EXOS-C), J. Geomag. Geoelectr., 37, 365-387, 1985.

OBARA, T. and H. OYA, Plasma conditions in the polar ionosphere observed in SPW experiments of PPS system on board the Ohzora (EXOS-C) satellite, J. Geomag. Geoelectr., 37, 285-307, 1985.

Oya, H., A. Morioka, and T. OBARA, Leaked AKR and terrestrial hectometric radiations discovered by the plasma wave and planetary plasma sounder experiments on board the Ohzora (EXOS-C) satellite--Instrumentation and observation results of plasma wave phenomena, J. Geomag. Geoelectr., 37, 237-262, 1985.

Pertie, L. E., Topside spread echoes, Can. J. Phys., 41, 194-195, 1963. 